and ; have no communication with the gastric lumen. GID are diagnosed most commonly in the first 2 years of age. Early diagnosis and surgical correction in the neonatal period usually are advocated to ovoid potential morbidity and mortality.

Case report In this report, we present a 3-days-old newborn with antenatal diagnosis of gastric duplication confirmed postnatally. It was successfully managed using open surgical resection. Histology confirmed the diagnosis. The postoperative course was unevent full. At 5 months, the infant had a good follow-up.

Conclusion Gastric duplications are very rare in newborns. Symptoms are atypical. Antenatal diagnosis is possible and allowed planning management. The treatment is based on a complete excision. Laparoscopic surgery is successful but needs to prove its safety and effectiveness.

\section{P643 RISK FACTORS FOR POSTOPERATIVE MORTALITY IN CONGENITAL DIAPHRAGMATIC HERNIA: A STUDY OF 29 CASES}

${ }^{1}$ Chiraz Regaieg*, 'Douha Kamoun, ${ }^{1}$ Rim Zaghdoud, ${ }^{2}$ Zitouni Hayet, ${ }^{1}$ Amel Ben Hamed, ${ }^{1}$ Manel Charfi, 'Amira Bouraoui, 'Nedia Hmida, ${ }^{2}$ Riadh Mhiri, ${ }^{1}$ Afef Ben Thabet, ${ }^{1}$ Abdellatif Gargouri. 'Department of Neonatology, Hedi Chaker Hospital, Sfax, Tunisia; ${ }^{2}$ Department of Pediatric Surgery, Hedi Chaker Hospital, Sfax, Tunisia

\subsection{6/archdischild-2019-epa.974}

Background Congenital diaphragmatic hernia (CDH) is one of the more common congenital anomalies with a frequency of $1 / 2200$ live births. Despite progress in antenatal diagnosis and neonatal care, mortality for $\mathrm{CDH}$ remains high close to 30$40 \%$. Several factors have been recognized as correlating with the prognosis of $\mathrm{CDH}$ such as pulmonary hypoplasia and pulmonary arterial hypertension. The aim of this study was to identify predictive factors of mortality after surgical management of $\mathrm{CDH}$.

Methods It is a retrospective study of all cases of $\mathrm{CDH}$ that were admitted at the neonatology department and were opered in the department of pediatric surgery in Sfax (Tunisia) from 2010 to 2018. The risk factors investigated were sex, prenatal diagnosis of $\mathrm{CDH}$, gestational age, birth weight, Apgar score, left side of $\mathrm{CDH}$, inhaled nitric oxide, vasoactive support, delay to surgery and surgical duration.

Results During the 9-year period, twenty nine patients were included. Of those nine were wright sides and twenty were left sides. $58.6 \%$ patients were male and $41.4 \%$ were female. The mean gestational age was $38.3 \%$. Six infants were premature. One newborn had a congenital heart disease. Antenatal diagnosis was performed in only $38 \%$ of cases $(n=11)$. Twelve newborns had low Apgar score. The mean hospital stay was 9, 6 days. Mortality rate was $62 \%$. There was no statistically significant difference between survival and death groups in terms of sex, prenatal diagnosis of $\mathrm{CDH}$, gestational age, left side of $\mathrm{CDH}$, inhaled nitric oxide and delay to surgery. However, low Apgar score and birth weight $<2700$ g were independently associated with postoperative mortality.

Conclusion Despite advances in neonatal reanimation, mortality for $\mathrm{CDH}$ remains high. Our study showed that low Apgar and low birth weight are risk factors for mortality.

\section{P644} MANAGEMENT OF CONGENITAL PULMONARY MALFORMATIONS: A REPORT ON 9 CASES

Amel Ben Hamed, Chiraz Regaieg*, Adel Babay, Manel Charfi, Amira Bouraoui, Nedia Hmida, Ridha Regaieg, Afef Ben Thabet, Abdellatif Gargouri. Department of Neonatology, CHU HEDI Chaker, Sfax, Tunisia

10.1136/archdischild-2019-epa.975

Background Congenital lung malformations (CLMs)) comprise a group of anatomical abnormalities of the respiratory tree. The most common of which include congenital cystic adenomatoid malformation (CAM), bronchopulmonary sequestration (PS), bronchial atresia, congenital lobar emphysema (CLE) and bronchogenic cyst. These anomalies are detected with increasing frequency by pre-natal sonography. When symptomatic, there is little controversy that resection is indicated, which is usually curative. When a lesion is asymptomatic there is greater debate regarding the benefit of resection versus continued observation. The aim of this study is to analyse the management options available and the moyen-term outcomes associated with each treatment option.

Methods We enrolled neonates who were admitted to the departement of neonatology at Hedi Chaker hospital, Sfax (Tunisia) within the ten last years and suffered from CLMs.

Results Nine cases were reviewed. Among these 8 were boys $(88 \%)$ and 3 neonates were preterm. The diagnosis was prenatal in 6 cases $(66 \%)$ and foetal sonography was abnormal in 8 cases (88\%). The adaptation to extrauterine life was good in 8 cases $(88 \%)$. Six infants $(66 \%)$ were symptomatic when admitted to neonatal unit. Radiological investigations ledeed to the diagnosis in all cases: 5 CAM, 3 PS and CLE in 1 case. Surgery was indicated for three patients. Two were operated before the age of 7 days due to severe clinical symptoms. The other patient was operated at the age of 7 years for possible malignant transformation of a PS. The surgical treatment involved a lobectomy for 2 patients and a thoracoscopy with malformation's in the other case. The histopathological examinations confirmed the diagnosis in all cases. Except for one patient with CAM, who died a few days after a lobectomy due to acute nosocomial pneumonia, the evolution was good for 8 children with a mean of follow-up of 24 months $(10$ months to 10 years).

Conclusion While the neonatal management of symptomatic CLMs is clear and includes prompt surgery, controversies remain for asymptomatic CPAM due to risk of infections and malignancies.

\section{P645 AN AUDIT TO IMPROVE NEONATAL READMISSION NUMBER AT WEXFORD GENERAL HOSPITAL}

Riaz Ahmed*, Naeem Shori, Allie Bone, Muhammad Jawad. Wexford general hospital, wexford, Ireland

\subsection{6/archdischild-2019-epa.976}

Introduction A readmission within a few weeks after discharge of an ostensibly healthy new-born from a well-baby nursery and postnatal ward is an undesirable event for parents, physicians, and payers. Such a readmission may reflect an inadequate assessment of the new-born's readiness for discharge, a lack of resources and/or an inability of a parent to provide early new-born care, or inappropriate and/or untimely availability of, or access to, outpatient care. Potentially preventable 
problems including jaundice and feeding problems account for the majority of hospital readmissions among new-borns within 28 days after discharge. Numerous studies have reported that the length of stay (LOS) for childbirth has been steadily decreasing in recent decades, in an effort to decrease costs and de medicalise pregnancy. The medical necessity of hospitalisation for and after childbirth is influenced by a variety of factors.

Aim To assess the preventable causes of neonatal readmission to Paediatric unit in and find a link with current practice of discharge from postnatal ward by comparing with practice followed at tertiary care maternity hospital across Ireland in one year.

Standard Protocols followed at three tertiary care maternity hospitals at Dublin Ireland

Nice guidelines of postnatal care

European Standards of New born care

Methodology Retrospective review of admission notes of neonates who were less than two weeks old admitted to WGH in 2018 from January to December, excluding babies transferred from other hospital.

Results A total of 24 babies less than two weeks of age were readmitted to Paediatric unit WGH which makes $1.4 \%$ of the total babies who were discharged from the postnatal unit. Among the 24 readmitted babies $37.5 \%$ were readmitted with Jaundice, while feeding issues and to out rule sepsis consisted of $20 \%$ each. The rest were minor causes like delayed passage of meconium, BRUE, transfer from SCBU.

Conclusion Jaundice and feeding issues are the two most important reason for the babies readmitted to Paediatric unit at Wexford general Hospital. In our audit it was found that all those babies who were readmitted from postnatal ward had no documented weight and bilirubin level check at the discharge.

Recommendations All those babies from postnatal ward should who serum bilirubin checked by transcutaneous billimeter and their weight measured at discharged especially the breast fed babies.

Loop audit After a period of 1 year following these recommendations.

\section{P646 CONGENITAL EPILUS OF ANTENATAL DIAGNOSIS: A CASE REPORT}

${ }^{1}$ Chiraz Regaieg*, ${ }^{1}$ Amel Ben Hmed, 'Abdelkrim Rim, ${ }^{1}$ Fathi Karray, 'Amira Bouraoui, ${ }^{1}$ Nedia Hmida, ${ }^{1}$ Ridha Regaieg, ${ }^{2}$ Mohamed Abdelmoula, ${ }^{1}$ Afef Ben Thabet, ${ }^{1}$ Abdellatif Gargouri. 'Department of Neonatology, Hedi Chaker Hospital, Sfax, Tunisia; ${ }^{2}$ Deptement of Maxillofacial Surgery, Habib Bourguiba, Sfax, Tunisia

10.1136/archdischild-2019-epa.977

Background Congenital epulis is a rare benign oral cavity tumor that usually arises from the maxillary alveolar mucosa. It is also known as congenital gingival granular cell tumor. This tumor can interfere with respiration and feeding. Prenatal diagnosis is uncommon and mostly confined to the third trimester.

Case report A 30-year-old woman, gravida 5, para 3, had an ultrasound examination at 30 weeks of gestation. A intraoral mass was noted to fill the fetus's oral cavity. Therefore, the woman was referred to our departement of neonatology, but she refused the complement of th explorations and the transfer. At 37 weeks of gestation, an elective cesarean section was performed. The newborn female child weighed $3 \mathrm{~kg}$ who had a large mass occupying the oral cavity. The mass prevented normal closure of the mouth and interfered with breastfeeding, but did not pose an immediate airway concern. She was referred to our departement immediately after birth. On clinical examination, a pedunculated mass, exhibiting a grey ulcerated surface was located on the left side of the maxillary alveolar ridge. This mass measured $6 \mathrm{~cm} \times 4.5 \mathrm{~cm}$ $\times 3 \mathrm{~cm}$ and prevented normal closure of the mouth and interfered with breast or bottle-feeding, but did not cause airway obstruction or respiratory distress. Examination of other systems was normal. A surgical excision of the mass was performed on the second day of neonatal life confirmed the presence of a tumor resembling epulis. The correctness of this diagnosis was subsequently confirmed by histogenesis. The intraoperative and postoperative courses were uneventful. The newborn recovered with no complications, and breastfeeding was initiated on the subsequent day of operation.

Conclusion Early diagnosis of CE in a newborn is of paramount importance in the successful management of these rare cases.

\section{P647 NEONATAL BRADYCARDIA AS PRESENTING SIGN OF CONGENITAL HYPOTHYROIDISM. CASE REPORT}

Margit Benke*, Raef Abouelhussein, Rizwan Khan, Niazy Al-Assaf. Neonatal Unit, University Maternity Hospital Limerick, Limerick, Ireland

\subsection{6/archdischild-2019-epa.978}

Introduction The incidence of congenital hypothyroidism is approximately 1:3500 babies worldwide, its more common in the Irish population (1:2300). Primary congenital hypothyroidism is the most common treatable cause of developmental delay, prompt diagnosis is important to prevent abnormal brain development and intellectual disability. Signs and symptoms of congenital hypothyroidism are rarely seen due to an early detection by the newborn bloodspot screening programme. Bradycardia is a very rare presentation of congenital hypothyroidism.

Case report A term male infant born by elective c-section was admitted to the neonatal unit from theatre following a dusky episode and respiratory distress. He required respiratory support with high flow nasal canula due to Transient Tachypnoea of the Newborn. During his admission a low baseline heart rate was noted around $100 / \mathrm{min}$ which became most pronounced on day 4 (HR: 65-80/min). Both ECHO and ECG revealed no cause of his bradycardia. $\mathrm{He}$ required phototherapy for DCT negative jaundice and nasogastric feeds for slow feeding. Hypothyroidism was suspected, both thyroid function tests and newborn bloodspot screening revealed severe congenital hypothyroidism (THS > $150 \mathrm{mU} / \mathrm{L}$, free T4: $4.6 \mathrm{pmol} / \mathrm{L}$ ). Technetium- $99 \mathrm{~m}$ pertechnetate thyroid scan confirmed agenesis of the thyroid gland. He was commenced on L-thyroxin therapy. His heart rate normalised and he established oral feeding within 48 hours of initiating treatment and he was discharged home well.

Conclusion Congenital hypothyroidism is mainly diagnosed by the newborn bloodspot screening programme. However, clinicians must keep an index of suspicion to the rare presentation of hypothyroidism in newborn babies to reach diagnosis as early as possible. 